\title{
Stokes operators, angular momentum and radiation phase
}

\section{Alexander S. Shumovsky \& Özgür E. Müstecaplioğlu}

To cite this article: Alexander S. Shumovsky \& Özgür E. Müstecaplioğlu (1998) Stokes operators, angular momentum and radiation phase, Journal of Modern Optics, 45:3, 619-628, DOI: $10.1080 / 09500349808231919$

To link to this article: http://dx.doi.org/10.1080/09500349808231919

$$
\text { 曲 Published online: } 03 \text { Jul } 2009 .
$$

Submit your article to this journal

Цll Article views: 61

Q View related articles $\asymp$

Citing articles: 3 View citing articles 
JOURNAL OF MODERN OPTICS, 1998, VOL. 45, NO. 3, 619-628

\title{
Stokes operators, angular momentum and radiation phase
}

\author{
ALEXANDER S. SHUMOVSKY and \\ ÖZGÜR E. MÜSTECAPLIOĞLU \\ Physics Department, Bilkent University, Bilkent, 06533 Ankara, \\ Turkey
}

(Received 6 May 1997; revision received 28 July 1997)

\begin{abstract}
We present a development of a method determining the quantum phase of radiation via the conservation of the angular momentum. It is shown that a set of generalized Stokes operators can be obtained with the aid of integrals of motion and two of them determine the radiation cosine and sine of the phase which corresponds to the azimuthal phase of the angular momentum according to the construction. We compare the evolution of the radiation cosine and sine and that for the conventional phase difference in the Jaynes-Cummings model. Although the expectation values coincide in some important cases, there is a striking difference between the behaviours of variances.
\end{abstract}

\section{Introduction}

In this paper we discuss the approach to quantum phase of light which has been proposed recently in [1]. We extend this approach to obtain the generalized Stokes operators of an electric dipole radiation. These operators correspond to the polar decomposition of two dual representations of the angular momentum [2]. Two of them can be interpreted as the Hermitian cosine and sine operators, corresponding to the azimuthal phase of the angular momentum. We compare our method with the classical description of the phase difference between the modes with different polarization and with some known approaches to quantum phase.

The problem of quantum phase has been widely studied in quantum optics (see, for recent reviews, [3-5]). One of the most important and popular methods in this field has been proposed by Pegg and Barnett [6-8]. They get over the difficulties connected with the definition of the Hermitian phase operator by introduction of a state space of formally finite dimension in the infinite Hilbert space. The infinite-dimension limit is taken only after the expectation values have been calculated. Within the framework of this approach, a number of interesting and important results have been obtained.

Another important way to describe the quantum phase properties of light is to examine what can be measured in a real experiment [9-11] (see also [12]). The cosine and sine of the phase operators identified in this approach correspond to the measured functions of the phase difference between two fields which can be considered as the modes with different polarizations [13]. These operational cosine and sine values should correspond to a unique intrinsic quantum-dynamical variable responsible for the phase properties of light [14]. The results of the operational approach [9-11] can be interpreted within the method based on the integration of the quasiprobability distribution function over the radial [15-19]. 
Exactly, the phase distribution measured by a homodyne detector is the radiusintegrated $Q$ function.

The approach has been proposed in [1] is complementary, in some sense, to the operational definition of the phase. It is based on a simple idea that the phase properties first are obtained by light in the process of generation and only then are measured in some way. This idea permits us to determine the quantum phase of radiation via the conservation laws, corresponding to the process of generation. Precisely, the conservation of the angular momentum should be examined because two other conserved quantities (energy and momentum) do not have non-trivial angular dependence.

The attempts to determine the phase as a quantum variable canonically conjugated with the angular momentum are well known (for example [20-23]). The new element is that we determine the quantum phase of radiation via the angular momentum of the source. In fact, the photon generated by an atomic transition takes away the angular momentum of this transition. The phase properties of the atomic transition moment can be easily determined by the polar decomposition [24] which is always possible in the finite dimensional space of the atomic states. At the same time, the $S U(2)$ subalgebra in the Weyl-Heisenberg algebra, describing the angular momentum of the radiation field, does not have a uniquely determined polar decomposition in the whole Hilbert space. Therefore, we determine the cosine and sine operators of the 'radiation phase' as the complements of corresponding atomic cosine and sine operators with respect to the integrals of motion, describing the conservation of total angular momentum [1].

It is well known from the quantum electrodynamics that the angular momentum (spin) of a photon determines its polarization [25]. In fact, radiation with given polarization is considered as a beam of photons with one and the same spin state. In classical optics, the polarization of light is specified by the Stokes parameters, which are usually considered for the two-mode field either in the linear polarization basis or in the circular polarization basis [26]. In the set of four Stokes parameters for the two-mode field, two of them determine the classical cosine and sine of the phase difference between the modes. The quantum conterpart of the Stokes parameters is provided by the Hermitian Stokes operators [27]. Recently the polar decomposition of the Stokes operators of the two-mode field has been examined [28-30]. In this approach, the Hermitian phase operator, describing the phase difference between two circularly polarized modes, is constructed under some special condition which is, in fact, equivalent to the consideration of a finite subspace of the Hilbert space with successive limit transition in the spirit of the Pegg-Barnett prescription. This phase-difference operator does not coincide with the difference between two Pegg-Barnett operators [30]. Let us stress that the consideration of more than one mode is important because the absolute phase of a single mode is not accessible for a measurement [10].

Since the minimum value of the angular momentum, which can be transmitted from the atomic transition to a photon, is equal to unity, let us consider, for simplicity, the electric dipole radiation. It is well known from the classical electrodynamics that, in this case, the magnetic field is always orthogonal to the direction of propagation while the electric field can have a component along the direction of propagation (at least, in the near zone) [31]. In the quantum domain, this radiation is described by the photons with given angular momentum $j=1$ and 
projection $m=0, \pm 1$ or, in other words, by the spherical photons [32]. Naturally, all three permissible projections, corresponding to the circularly polarized components with the opposite helicities and one linearly polarized component, should be taken into consideration. Although the intensity of the linearly polarized component with $m=0$ is extremely small in the far zone, it is not surprising that it must be taken into account in the quantum domain. Even if this component is supposed to be in the vacuum state, it can contribute to the quantum fluctuations. Corresponding classical polarization properties are described by the $3 \times 3$ tensor of polarization. Since the number of independent parameters is equal to five in this case (the intensities of three components with different $m$ and the phase differences $\Delta_{m m^{\prime}}$ such that $\Delta_{+0}+\Delta_{0-}+\Delta_{-0}=0$ ), the set of the generalized Stokes parameters should consist of five independent parameters. Thus, in the quantum domain, we have to consider five generalized Stokes operators.

Below in this paper we show how the generalized Stokes operators can be determined via the conservation of the angular momentum in an atom-field interaction. In this way, we determine the quantum phase properties of the electric dipole radiation in terms of the Hermitian cosine and sine operators. According to the construction, these operators describe the azimuthal phase of the angular momentum. Under some special conditions, this phase could coincide with the phase difference between two components of the electric dipole radiation. We compare our results with that obtained within some known approaches.

\section{Phase properties of classical electric dipole radiation}

The Stokes parameters of classical electromagnetic field determine the relative phases of two components with different polarization [26]. In the circular polarization basis, the definitions are

$$
\begin{aligned}
& s_{0}=\left|\boldsymbol{\epsilon}_{+} \cdot \mathbf{E}\right|^{2}+\left|\boldsymbol{\epsilon}_{-} \cdot \mathbf{E}\right|^{2}, \\
& s_{1}=2 \operatorname{Re}\left[\left(\boldsymbol{\epsilon}_{+} \cdot \mathbf{E}\right)^{*}\left(\boldsymbol{\epsilon}_{-} \cdot \mathbf{E}\right)\right], \\
& s_{2}=2 \operatorname{Im}\left[\left(\boldsymbol{\epsilon}_{+} \cdot \mathbf{E}\right)^{*}\left(\boldsymbol{\epsilon}_{-} \cdot \mathbf{E}\right)\right], \\
& s_{3}=\left|\boldsymbol{\epsilon}_{+} \cdot \mathbf{E}\right|^{2}-\left|\boldsymbol{\epsilon}_{-} \cdot \mathbf{E}\right|^{2},
\end{aligned}
$$

where $\mathbf{E}$ is the field and $\boldsymbol{\epsilon}$ is the unit vector of polarization. Thus, the parameters $s_{1}$ and $s_{2}$ determine cosine and sine of the phase difference between two circularly polarized components.

In view of our aim, let us consider the multipole expansion of the field provided by the expression [31]

$$
\mathbf{A}=\sum_{k \lambda j m}\left[a_{\lambda j m}(k) \mathbf{A}_{\lambda j m}(k)+\mathrm{cc}\right], \quad \mathbf{E}=\sum_{k \lambda j m}\left[a_{\lambda j m}(k) \mathbf{E}_{\lambda j m}(k)+\mathrm{cc}\right],
$$

where $\mathbf{E}_{\lambda j m}(k)=\mathrm{i} k \mathbf{A}_{\lambda j m}(k)$, the index $\lambda=E, M$ shows the type of the multipole field and

$$
a_{\lambda j m}(k) \equiv \int r^{2} \mathrm{~d} r \mathrm{~d} \Omega \mathbf{E}_{\lambda j m}^{*}(k) \cdot \mathbf{E}
$$

Here the coefficients $\mathbf{A}_{\lambda j m}(k)$ are defined by the standard combinations of vector spherical harmonics and Bessel functions (for example [31, 32]). Since the 
standard Stokes parameters (1) are determined by a quadratic function of the field which does not contain the rapidly oscillating terms [26], let us consider the form

$$
\left[\mathbf{E}_{\lambda j m}^{*}(k) \cdot \mathbf{E}\right]^{*}\left[\mathbf{E}_{\lambda j n}^{*} \cdot \mathbf{E}\right] \equiv \rho_{m n}
$$

where the vector $\mathbf{E}$ is written in the 'phasor' form, dropping the conjugated part. Since we are primarily interested in the electric dipole radiation, we may restrict our consideration by the case of $\lambda=E, j=1$ and $m=0, \pm 1$. Then the modes $m= \pm 1$ describe two circularly polarized components with the opposite helicities and $m=0$ corresponds to a linearly polarized mode which always exists in the classical dipole radiation. The $3 \times 3$ Hermitian matrix (3) can be represented as

$$
\rho=\mathcal{N}+\Lambda+A^{+}
$$

where the elements of the diagonal matrix $\mathcal{N}$ clearly are the intensities of modes with different $m$. The elements of the triangular matrix $\boldsymbol{A}$ are such that

$$
\operatorname{Re}\left(\Lambda_{m n}\right) \propto \cos \Delta_{m n}, \quad \operatorname{Im}\left(\Lambda_{m n}\right) \sim \sin \Delta_{n m}
$$

where $\Delta_{m n} \equiv \arg \left(\mathbf{E}_{m}^{*} \cdot \mathbf{E}\right)-\arg \left(\mathbf{E}_{n}^{*} \cdot \mathbf{E}\right)^{*}$. It should be stressed that, unlike the case of standard Stokes parameters (1), there are three phase differences $\Delta_{m n}$, only two of which are independent because of the equality $\Delta_{+-}=\Delta_{+0}-\Delta_{-0}$. To specify the components of $\mathcal{N}$, let us introduce the following combinations:

$$
\begin{aligned}
& \mathcal{S}_{3}=\frac{1}{2}\left[2\left|\mathbf{E}_{0}^{*} \cdot \mathbf{E}\right|^{2}-\left|\mathbf{E}_{+}^{*} \cdot \mathbf{E}\right|^{2}-\left|\mathbf{E}_{-}^{*} \cdot \mathbf{E}\right|^{2}\right], \\
& \mathcal{S}_{4}=\frac{3^{1 / 2}}{2}\left[\left|\mathbf{E}_{-}^{*} \cdot \mathbf{E}\right|^{2}-\left|\mathbf{E}_{+}^{*} \cdot \mathbf{E}\right|^{2}\right] .
\end{aligned}
$$

It is clear that, when the intensity of the linearly polarized component vanishes, the parameters $\mathcal{S}_{3}$ and $\mathcal{S}_{4}$, apart from the constant multipliers, are just the Stokes parameters $s_{0}$ and $s_{3}$ respectively in equation (1). The reason to choose the constant factors will be clarified in section 4 . Two more relations, specifying the components of $\Lambda$ and determining the possible phase difference, can be chosen as follows:

$$
\begin{aligned}
& \mathcal{S}_{1}=\operatorname{Re}\left[\left(\mathbf{E}_{+}^{*} \cdot \mathbf{E}\right)^{*}\left(\mathbf{E}_{0}^{*} \cdot \mathbf{E}\right)+\left(\mathbf{E}_{0}^{*} \cdot \mathbf{E}\right)^{*}\left(\mathbf{E}_{-}^{*} \cdot \mathbf{E}\right)+\left(\mathbf{E}_{-}^{*} \cdot \mathbf{E}\right)^{*}\left(\mathbf{E}_{+}^{*} \cdot \mathbf{E}\right)\right], \\
& \mathcal{S}_{2}=\operatorname{Im}\left[\left(\mathbf{E}_{+}^{*} \cdot \mathbf{E}\right)^{*}\left(\mathbf{E}_{0}^{*} \cdot \mathbf{E}\right)+\left(\mathbf{E}_{0}^{*} \cdot \mathbf{E}\right)^{*}\left(\mathbf{E}_{-}^{*} \cdot \mathbf{E}\right)+\left(\mathbf{E}_{-}^{*} \cdot \mathbf{E}\right)^{*}\left(\mathbf{E}_{+}^{*} \cdot \mathbf{E}\right)\right] .
\end{aligned}
$$

It is clear that $2 \mathcal{S}_{1}=s_{1}$ and $2 \mathcal{S}_{2}=s_{2}$ when the linearly polarized component vanishes. Thus, the set of four parameters (4), (5) can be considered as some generalization of (1) in the case of the electric dipole radiation. It should be emphasized that the total set of the Stokes parameters, describing polarization properties of electric dipole radiation, should consist of five parameters. The additional parameter can be specified as $\operatorname{Tr} \mathcal{N}$. The quantum counterpart of the parameters (4), (5) can be obtained by substitution of the operators of spherical photons into equations (2) and (3) instead of the complex amplitudes $a_{m}$ in a 
standard way $[32,33]$. Then we get the generalized Stokes operators

$$
\begin{aligned}
& S_{0}=\sum_{m=-1}^{+1} a_{m}^{+} a_{m} \equiv N, \\
& S_{1}=\frac{1}{2}\left[\left(a_{+}^{+}+a_{-}^{+}\right) a_{0}+a_{-}^{+} a_{+}+\mathrm{hc}\right], \\
& S_{2}=\frac{1}{2}\left[\left(a_{+}^{+}-a_{-}^{+}\right) a_{0}+a_{-}^{+} a_{+}-\mathrm{hc}\right], \\
& S_{3}=\frac{1}{2}\left(2 a_{0}^{+} a_{0}-\sum_{m= \pm 1} a_{m}^{+} a_{m}\right), \\
& S_{4}=\frac{3^{1 / 2}}{2}\left(a_{-}^{+} a_{-}-a_{+}^{+} a_{+}\right) .
\end{aligned}
$$

Clearly, these are Hermitian operators such that

$$
\left[S_{1}, S_{2}\right]=\left[S_{1}, s_{0}\right]=\left[S_{2}, S_{0}\right]=\left[S_{3}, S_{0}\right]=\left[S_{4}, S_{0}\right]=0 .
$$

Let us also note that, unlike the standard Stokes parameters $s_{1}$ and $s_{2}$ in equation (1), the parameters $\mathcal{S}_{1}$ and $\mathcal{S}_{2}$ in equation (5) do not have the simple meaning of the cosine and sine of a phase difference. However, as we are going to see, they correspond to the cosine and sine of some quantum phase variable.

\section{Polar decomposition of the angular momentum}

The angular momentum of the electric dipole radiation is determined by the operators [32]

$$
M_{z}=\sum_{m=-1}^{+1} m a_{m}^{+} a_{m}, \quad M_{+}=2^{1 / 2}\left(a_{+}^{+} a_{0}+a_{0}^{+} a_{-}\right), \quad M_{-}=2^{1 / 2}\left(a_{0}^{+} a_{+}+a_{-}^{+} a_{0}\right),
$$

forming a representation of the $S U(2)$ subalgebra in the Weyl-Heisenberg algebra. One can expect to determine the quantum phase properties of the angular momentum through the use of the polar decomposition

$$
M_{+}=\left(M_{+} M_{-}\right)^{1 / 2} \exp (\mathrm{i} \hat{\phi}),
$$

similar to that proposed by Dirac [34]. Unfortunately, the exponential of the phase operator in equation (9) cannot be uniquely determined as a unitary operator in the whole Hilbert space. The reason is that the subalgebra (8) does not contain a uniquely determined scalar (the Casimir operator) in the enveloping algebra. Therefore, in the spirit of the philosophy of [1], we determine this polar decomposition via the conservation of the angular momentum in the atom-field interaction. Consider for simplicity the Jaynes-Cummings model describing the electric dipole radiation in a spherical box of volume $V$ (see [35] and references therein)

$$
H=\sum_{m}\left[\omega\left(a_{m}^{+} a_{m}+R_{m m}\right)+\mathrm{i} g\left(R_{m G} a_{m}-a_{m}^{+} R_{G m}\right)\right], \quad g=D\left(\frac{c^{4} \omega}{\hbar V}\right)^{1 / 2} .
$$

Here $D$ is the effective dipole factor, $R_{\alpha \beta}$ are the atomic operators describing the transitions between the excited sublevels $j=1 ; m=0, \pm 1$ and ground level $G$ with $j_{G}=0 ; m_{G}=0$. Then the generators of the $S U(2)$ algebra, specifying the atomic angular momentum $j=1$, are expressed in terms of the atomic operators as 
follows:

$$
J_{z}=R_{++}-R_{--}, \quad J_{+}=2^{1 / 2}\left(R_{+0}+R_{0-}\right), \quad J_{-}=2^{1 / 2}\left(R_{0+}+R_{-0}\right) .
$$

It is not hard to see that the operators $J_{\alpha}+M_{\alpha}$ commute with the Hamiltonian (10), owing to conservation of the total angular momentum. Since the enveloping algebra of equation (11) contains the Casimir operator $\mathrm{J}^{2}=2 \times 1=2 \sum_{m} R_{m m}$, the polar decomposition of the form (9) can be determined [24] by choosing the exponential of the phase operator and corresponding sine and cosine operators as follows:

$$
\begin{aligned}
\mathcal{E} & =R_{+0}+R_{0_{-}}+R_{-+}, \quad \mathcal{E} \mathcal{E}^{+}=1, \quad \mathcal{E}^{3}=\mathcal{E}, \\
S_{\mathrm{A}} & =\frac{\mathcal{E}-\mathcal{E}^{+}}{2 \mathrm{i}}, \quad C_{\mathrm{A}}=\frac{\mathcal{E}+\mathcal{E}^{+}}{2} .
\end{aligned}
$$

Clearly, $S_{\mathrm{A}}$ and $C_{\mathrm{A}}$ are the Hermitian commuting operators such that $S_{\mathrm{A}}^{2}+$ $C_{\mathrm{A}}^{2}=1$. They are determined in terms of the atomic operators in the basis formed by the states $|j=1 ; m=0, \pm 1\rangle$. It is easily seen that, because of the conservation laws $\left[S_{\mathrm{A}}+S_{2}, H\right]=0$, one can interpret the operators $C_{\mathrm{R}}=S_{1}$ and $S_{\mathrm{R}}=S_{2}$ as the radiation counterparts of $C_{\mathrm{A}}$ and $S_{\mathrm{A}}$.

In addition to the above basis of the atomic states, one can choose the orthogonal states $\left|\varphi_{m}\right\rangle, \varphi_{m}=2 m \pi / 3$ such that $\mathcal{E}\left|\varphi_{m}\right\rangle=\exp \left(i \varphi_{m}\right)\left|\varphi_{m}\right\rangle$. Employing these states then gives the dual representation of the algebra (11) [24]:

$$
\Phi_{z}=\sum_{m=-1}^{+1} m\left|\varphi_{m}\right\rangle\left\langle\varphi_{m}\left|, \quad \Phi_{+}=\sum_{m=-1}^{+1}[2-m(m+1)]^{1 / 2}\right| \varphi_{m+1}\right\rangle\left\langle\varphi_{m}\right|, \quad \Phi_{-}=\Phi_{+}^{+} .
$$

By performing a polar decomposition of this algebra, one can obtain the atomic 'sine' and 'cosine' operators in the $\Phi$ representation [2]:

$$
S_{\mathrm{A} \Phi}=-\frac{3^{1 / 2}}{2}\left(R_{++}-R_{--}\right), \quad C_{\mathrm{A} \Phi}=R_{00}-\frac{1}{2}\left(R_{++}+R_{--}\right),
$$

which clearly are the atomic counterparts of the generalized Stokes operators $S_{3}$ and $S_{1}$ respectively, because $\left[S_{\mathrm{A} \Phi}+S_{3}, H\right]=\left[C_{\mathrm{A} \Phi}+S_{1}, H\right]=0$. In addition, $\left[S_{0}, \mathbf{J}^{2}\right]=0$. Thus, the use of the polar decomposition of the atomic angular momentum and conservation of the total angular momentum in the system 'atom + radiation' leads to the direct definition of the generalized Stokes operators (6).

\section{Radiation sine and cosine of the phase operators}

Since the operators $S_{1}$ and $S_{2}$ in equation (6) are the counterparts of the atomic operators $C_{\mathrm{A}}$ and $S_{\mathrm{A}}$, one can choose to interpret them as the cosine and sine of the azimuthal phase of the radiation angular momentum. Below they will be termed the radiation cosine and sine respectively. In this case, they must be normalized in some way to have the expectation values between -1 and +1 . A convenient form of the radiation cosine and sine operators is afforded by taking [1]

$$
C_{\mathrm{R}}=K S_{1}, \quad S_{\mathrm{R}}=K S_{2},
$$

and requiring that $K$ is the real normalization factor. In the case of the JaynesCummings model (10), $K=1$ owing to the conservation laws. However, if we 
examine an unspecified source of the electric dipole radiation, $K$ should be considered as a function of intensities [2]. It seems to be quite natural to determine $K$ in this case by the condition

$$
\left\langle C_{\mathrm{R}}^{2}+S_{\mathrm{R}}^{2}\right\rangle=1
$$

which, in view of equations (6) and (12) can be put in the form

$$
\begin{aligned}
K= & {\left[I_{+}+I_{0}+I_{-}+I_{+} I_{0}+I_{0} I_{-}+I_{+} I_{-}\right.} \\
& \left.+\left\langle a_{+}^{+2} a_{0} a_{-}+a_{0}^{+2} a_{+} a_{-}+a_{-}^{+2} a_{+} a_{0}+\mathrm{hc}\right\rangle\right]^{2},
\end{aligned}
$$

where $I_{m} \equiv\left\langle a_{m}^{+} a_{m}\right\rangle$. Let us stress that, if we try to determine the classical cosine and sine

$$
\mathcal{C}_{\mathrm{R}}=\mathcal{K} \mathcal{S}_{1}, \quad \mathcal{S}_{\mathrm{R}}=\mathcal{K} \mathcal{S}_{2}, \quad \mathcal{C}_{\mathrm{R}}^{2}+\mathcal{S}_{\mathrm{R}}^{2}=1,
$$

through the use of equations (5), we get the constant $\mathcal{K}$, coinciding with equation (14) to within the linear term $\sum_{m} I_{m}$. This term follows from the commutation relations for the photon operators and is negligible in the classical limit.

It is not hard to see that the definitions (12) and (13) of the cosine and sine operators are consistent with the principle of uniform phase distribution in the number and vacuum states [2]. Moreover, $\left\langle C_{\mathrm{R}}\right\rangle=\left\langle S_{\mathrm{R}}\right\rangle=0$ and corresponding variances are equal to $\frac{1}{2}$ if only two modes out of three are in the number state. When all three modes are in the coherent states, the definitions (12) and (13) lead to the standard classical limit.

\section{Radiation phase and conventional phase difference}

Although the operators $S_{1}$ and $S_{2}$ in equation (6) or $C_{\mathrm{R}}$ and $S_{\mathrm{R}}$ in equation (12) describe the azimuthal phase of the angular momentum, in a special case when one of the modes of radiation is in the vacuum state, they formally correspond to the phase difference between two other modes. To make a simple comparison with the conventional definition of the phase difference, let us consider the evolutions described by the Jaynes-Cummings model (10). With the assumption that the atom is prepared initially in the superposition of two sublevels of the excited level $\left(p_{+}|j=1 ; m=+1\rangle+p_{-} \mid j=1 ; m=-1\right),\left|p_{+}\right|^{2}+|p|^{2}=1$ while the field is in the vacuum state, the time-dependent wavefunction can be written as

$$
\begin{aligned}
|\Psi(t)\rangle= & \cos (g t)\left(p_{+}|j=1 ; m=+1\rangle+p_{-}|j=1 ; m=-1\rangle\right)|0,0,0\rangle \\
& +\sin (g t)|j=0 ; m=0\rangle\left(p_{+}|1,0,0\rangle+p_{-}|0,0,1\rangle\right) .
\end{aligned}
$$

In this case, only two circularly polarized components with the opposite helicities can be emitted or absorbed while the linearly polarized component is kept in the vacuum state. Averaging the operators (6) with respect to the wavefunction (14) and taking into account the definition (12), we get

$$
\left\langle C_{\mathrm{R}}\right\rangle=\left|p_{+} p_{-}\right| \cos \Delta \sin ^{2}(g t), \quad\left\langle S_{\mathrm{R}}\right\rangle=\left|p_{+} p_{-}\right| \sin \Delta \sin ^{2}(g t),
$$

where $\Delta \equiv \arg p_{+}-\arg p_{-}$. Thus, the expectation values of the 'radiation' cosine and sine (12) can be interpreted, in the case under consideration, as the cosine and sine of the phase difference between two circularly polarized components. In other words, the averages (16) have the meaning of the Stokes parameters $s_{1}$ and $s_{2}$ in 
equation (1). The phase difference here is completely determined by the choice of the initial atomic state. For the variances we get

$$
\begin{aligned}
& V\left(C_{\mathrm{R}}\right)=\left[\frac{1}{2}\left(1+\left|p_{+} p_{-}\right| \cos \Delta\right)-\left|p_{+} p_{-}\right|^{2} \cos ^{2} \Delta \sin ^{2}(g t)\right] \sin ^{2}(g t), \\
& V\left(S_{\mathrm{R}}\right)=\left[\frac{1}{2}\left(1-\left|p_{+} p_{-}\right| \cos \Delta\right)-\left|p_{+} p_{-}\right|^{2} \sin ^{2} \Delta \sin ^{2}(g t) \sin ^{2}(g t) .\right.
\end{aligned}
$$

Let us stress that, although the linearly polarized component is in the vacuum state, the terms with $a_{0}$ and $a_{0}^{+}$in $C_{\mathrm{R}}$ and $S_{\mathrm{R}}$ contribute to the variances (17). It seems to be natural because the vacuum fluctuations of any mode $m$ of the cavity field cannot be eliminated.

Consider now th Pegg-Barnett phase difference determined for the modes with the opposite helicities in the system (10). The phase sums and differences have been determined in [36]. In this formalism, the phase properties of a two-mode field are simply constructed from the single-mode phase. However, the direct use of the individual phase definition [6-8] leads to the values of the phase difference, covering the $4 \pi$ range, and the phase difference should be cast into the $2 \pi$ range [36]. It was emphasized in [36] that there are many ways to apply the casting procedure. Below we use this procedure in the form proposed in $[28,37]$. The relation between this casting procedure and that by Barnett and Pegg [36] has been discussed in $[5,28,37]$. Then, the phase distribution over the relative phase $\phi=$ $\phi_{+}-\phi_{-}$in the range $2 \pi$ is

$$
P=\sum_{n=0}^{\infty}\left|\left\langle\phi^{(n)} \mid \psi\right\rangle\right|^{2}, \quad\left|\phi^{(n)}\right\rangle=\frac{1}{(2 \pi)^{1 / 2}} \sum_{n_{+}=0}^{n} \exp \left(\mathrm{i} \phi n_{+}\right)\left|n_{+}, 0, n-n_{+}\right\rangle .
$$

Then, the expectation value of any function of the relative phase is determined as follows:

$$
\langle F(\phi)\rangle=\int_{-\pi}^{\pi} P(\phi) F(\phi) \mathrm{d} \phi
$$

Inserting the function (15) as $|\psi\rangle$ into equation (18), we get

$$
\left\langle\cos \Phi_{\mathrm{PB}}\right\rangle=\left|p_{+} p_{-}\right| \cos \Delta \sin ^{2}(g t), \quad\left\langle\sin \Phi_{\mathrm{PB}}\right\rangle=\left|p_{+} p_{-}\right| \sin \Delta \sin ^{2}(g t)
$$

Thus, for the system with Hamiltonian (10), the expectation values of the 'radiation' cosine and sine have the same values at all times as the corresponding Pegg-Barnett expressions. In turn, the variances are

$$
\begin{aligned}
& V\left(\cos \Phi_{\mathrm{PB}}\right)=\left(\frac{1}{2}-\left|p_{+} p_{-}\right|^{2} \cos ^{2} \delta \sin ^{2}(g t)\right] \sin ^{2}(g t), \\
& V\left(\sin \Phi_{\mathrm{PB}}\right)=1-\left(\frac{1}{2}+\left|p_{+} p_{-}\right|^{2} \sin ^{2} \delta \sin ^{2}(g t)\right] \sin ^{2}(g t) .
\end{aligned}
$$

Unlike the average cosine and sine, the variances (17) and (20) are different although

$$
V\left(C_{\mathrm{R}}\right)+V\left(S_{\mathrm{R}}\right)=V\left(\cos \Phi_{\mathrm{PB}}+V\left(\sin \Phi_{\mathrm{PB}}\right)=1-\left|p_{+} p_{-}\right|^{2} \sin ^{4}(g t) .\right.
$$

This difference arises for the following reason. In the Pegg-Barnett approach, the cosine and sine (19) are determined in terms of the phase difference between two modes. Although the third mode could be included in the definition of the phase distribution function (18), it does not contribute to the quantum fluctuations. On the contrary, the radiation cosine and sine (12) describe the quantum phase properties of the angular momentum (spin) of a photon. They formally coincide 
with those, determined in the Pegg-Barnett approach, only in the case when the third mode is in the vacuum state. At the same time, they describe a different physical variable and take into account the vacuum fluctuations of all modes of the cavity field. Let us also note that, constructing the Stokes operators directly from equations (1) and using the polar decomposition in the spirit of [26-28], one can determine some other cosine and sine operators. These operators also determine the phase difference between two circularly polarized modes and formally differ from the objects of the Pegg-Barnett approach. Nevertheless, they also do not take into account of the vacuum fluctuations of the complete cavity field.

\section{Conclusions}

In this paper we have concentrated on the description of the quantum phase properties of radiation via the conservation of the angular momentum in the atomfield system. This way permits us to avoid the difficulties related to the polar decompositions in the whole Hilbert space of the photon states. The 'radiation phase' determined in this way in the Jaynes-Cummings model (10) is the azimuthal phase of the photon spin. It is shown that the use of the angular momentum conservation leads to the definition of the set of generalized Stokes operators equivalent to that obtained via the standard consideration of the classical polarization tensor with the consequent quantization. This set (6) completely determines the quantum polarization properties of the electric dipole radiation.

It follows from the results of section 3 that the atomic phase operator can be directly determined as follows:

$$
\Phi_{\mathrm{A}}=\frac{1}{3} \sum_{m=-1}^{+1} \varphi_{m}\left[\exp \left(-\mathrm{i} \varphi_{m}\right) \mathcal{E}+\exp \left(\mathrm{i} \varphi_{m}\right) \mathcal{E}^{+}\right], \quad \varphi_{m}=\frac{2 m \pi}{3}
$$

Then, the radiation counterpart clearly is $\Phi_{\mathrm{R}}=\left(4 \pi / 3^{1 / 2}\right) S_{2}$. Although for the atomic subsystem

$$
S_{\mathrm{A}}=\sin \Phi_{\mathrm{A}}, \quad C_{\mathrm{A}}=\cos \Phi_{\mathrm{A}}
$$

similar equalities do not occur in the radiation subsystem. Since the operators $S_{1}$ and $S_{2}$ (equation (6)) and $C_{\mathrm{R}}$ and $S_{\mathrm{R}}$ (equation (12)) have quite natural physical meaning, it seems to be unreasonable to consider $\Phi_{R}$ as the radiation phase.

Let us emphasize the principal difference between our approach and those based on the consideration of a phase operator for a one-mode field or phasedifference operators for two modes. The quantum phase properties, determined by the operators (6), are the intrinsic properties of a photon, related to its spin (generally, to its angular momentum). In the multiphoton case, they are determined by the mutual phase properties of all three allowed modes of radiation rather than the single-mode phase or the two-mode phase difference. In the special case of only two modes having non-zero intensities, the operators (12) determine the cosine and sine of the phase difference but only formally. In this case, the expectation values of (12) coincide with the standard mean cosine and sine of the phase difference between two modes. At the same time, the variance of cosine and sine are different because they correspond to different physical objects.

Since the radiation cosine and sine (12) commute with each other and with the total photon number, all of them can be measured at once. Since the polarization 
properties of radiation can be directly measured, the generalized Stokes parameters, which are the expectation values of the generalized Stokes operators, can be used to determine the measuring radiation phase in the operational way. In view of the above results, it is natural to suppose that the phase of the angular momentum of radiation might be considered as the intrinsic phase variable in the spirit of [14].

Although we have considered the results for the Jaynes-Cummings model (10), the approach will in principle work for an arbitrary system and even for an arbitrary state of the field generated by an unspecified source, in which case the definition (12) must be added by the normalization condition (13). This definition is compatible with the classical definition of the radiation cosine and sine in terms of the generalized Stokes parameters (6). It also shows the uniform quantum phase distribution in the vacuum and number states.

\section{References}

[1] Shumovsky, A. S., 1997, Optics Commun., 136, 219.

[2] Shumovsky, A. S., and Müstecapliớlu, Ö. E., 1997, Phys. Lett. $A$ (to be published).

[3] 1993, Physica Scripta T, 48.

[4] LynCH, R., 1995, Phys. Rep., 256, 367.

[5] 'Tanas, R., Miranowicz, A., and Gantsog, Ts, 1996, Prog. Optics, 35, 1.

[6] Pegg, D. T., and BarnetT, S. M., 1988, Europhys. Lett., 6, 483.

[7] BarnetT, S. M., and PegG, D. T., 1989, J. mod. Optics, 36, 7.

[8] Pegg, D. T., and Barnett, S. M., 1992, J. mod. Optics, 39, 2121.

[9] Noh, J. W., Fougéres, A., and Mandel, L., 1991, Phys. Rev. Lett., 67, 1426.

[10] Noh, J. W., Fougéres, A., and Mandel, L., 1992, Phys. Rev. A, 45, 242.

[11] Noh, J. W., Fougéres, A., and Mandel, L., 1993, Phys. Rev. A, 48, 1719.

[12] Barnett, S. M., and Pegc, D. T., 1993, Phys. Rev. A, 47, 4537.

[13] Накıö́lu, T., Shumovsky, A. S., and Aytür, O., 1994, Phys. Lett. A, 149, 304.

[14] Englert, B.-G., and Wodkiewicz, J., 1995, Phys.Rev. A, 51, R266.

[15] Bandilla, A.,, and Paul, H., 1969, Annln Phys,, , 323.

[16] Schleih, W. P., Bandilla, A., and Paul, H., 1992, Phys. Rev. A, 45, 6652.

[17] Freyberger, M., Vogel, K., and Schleich, W. P., 1993, Phys. Lett. A, 176, 41.

[18] Freyberger, M., and Schleich, W. P., 1993, Phys. Rev. A, 47, R30.

[19] Leonhard', U., and Paul, H., 1993, Phys. Rev. A, 47, R2460.

[20] Levi-Leblond, J. M., 1979, Ann. Phys. (N.Y.), 101, 319.

[21] Barnett, S. M., and PegG, D. T., 1990, Phys. Rev. A, 41, 3427.

[22] SÁnchez-Soto, L. L., and Luis, A., 1994, Optics Commun., 105, 84.

[23] Lukš, A., and Peřinova, V., 1994, Quant. Optics, 6, 125.

[24] Vourdas, A., 1990, Phys. Rev. A, 41, 1653.

[25] Berestetskil, V. B., Lifshitz, E. M., and Pitaevskit, L. P., 1982, Quantum Electrodynamics (Oxford: Pergamon).

[26] Born, M., and Wolf, E., 1970, Principles of Optics (Oxford: Pergamon).

[27] Jauch, J. M., and Rohrlich, F., 1959, The Theory of Photons and Electrons (Reading, Massachussetts: Addison-Wesley).

[28] Luis, A., and Sanchez-Soto, L. L., 1993, Phys. Rev. A, 48, 4702.

[29] Luis, A., and Sánchez-Soto, L. L., 1994, Optics Commun., 1, 84.

[30] Luis, A., Sánchez-Soto, L. L., and Tanaś, R., 1995, Phys. Rev. A, 51, 1634.

[31] JACKson, J. D., 1975, Classical Electrodynamics (New York: Wiley).

[32] Heitler, W., 1984, The Quantum Theory of Radiation, third edition (New York: Dover Publications).

[33] Davydov, A. S., 1976, Quantum Mechanics (Oxford: Pergamon).

[34] Duirac, P. A. M., 1927, Proc. R. Soc., 114, 243, 710.

[35] Rupasov, V. I., and Singh, M., 1966, Phys. Rev. A, 54, 3614.

[36] Barnett,S. M., and Pegg, D. T., 1990, Phys. Rev. A, 42, 6713.

[37] Tanas, R., and Gantsog, Ts., 1992, Optics Commun., 87, 369. 